\title{
Evaluation of Early Postoperative Cognitive Dysfunction Incidence and Involved Neurocognitive Functions in Patients with Cardiac and Noncardiac Surgery Under General Anesthesia
}

\author{
Carmen Sircuța ${ }^{\star *}$, Tunde Lucza ${ }^{2}$, Mihaly Veres ${ }^{1}$, Ildiko Szomoru ${ }^{3}$, Leonard Azamfirei ${ }^{1}$ \\ 1 University of Medicine and Pharmacy Tirgu Mures, Romania \\ 2 Emergency Institute for Cardiovascular Diseases and Transplantation Tirgu Mures, Romania \\ ${ }^{3}$ Emergency Clinical County Hospital of Tirgu Mures, Romania
}

\begin{abstract}
Objective: To analyse postoperative cognitive dysfunction's (POCD) incidence and cognitive areas involved, in patients with cardiac and general surgery. Material and Methods: Prospective observational study on 130 patients undergoing general or heart surgery on cardiopulmonary bypass, under general anesthesia. Two groups, 65 members each. Group A had a heart surgery and group B a noncardiac surgery. The same type of anesthetic drugs were used. All patients completed the Montreal Cognitive Assesment (MoCA) questionnaire: preoperative, 24 hours after stopping any medicine acting on central nervous system and 7 days postoperative. We compared the MoCA scores obtained on different cognitive domains in this moments for each group of patients, for neurocognitive functions: visuo-spatial executive, naming, attention, verbal fluency, abstraction, recall, orientation, final score. We compared the scores between the two groups at 24 hours and 7 days postoperatively for the same domains. Results: POCD was found at 24 hours testing in both groups. At 7 days postoperatively POCD was not found in any of the groups. There was no statistically significant difference in total final score between two groups at 24 hours nor at 7 days postoperative testing. There are significant differences between the two groups, with lower score in cardiac group in 5 of 7 fields at 24 hours testing, with the persistence of difference in 2 of 7 fields at 7 days. Conclusions: Overall POCD was present at 24 hours but was not found at 7 days testing for none of the groups. POCD is present in some neurocognitive domains and this depends on surgery type.
\end{abstract}

Keywords: POCD, neurocognitive domain, cardiovascular surgery, noncardiac surgery, general ansthesia

Received 21 January 2017 / Accepted 24 April 2017

\section{Introduction}

The postoperative neurocognitive dysfunction definition emerged about mid-1990s when it began to be described as a decline in cognitive performance. This postoperative decline which has to come out at least in two or three tests of a battery especially created for this aim [1].

Nowadays postoperative cognitive dysfunction is a well known perioperative syndrome, accounting for about $15 \%$ in patients older than 60 years old, when a proven decrease in cognitive function appears. This postoperative reduction is proven by objective testing and is due to anesthesia and surgery, without being able to precisely know the exact cause of it [2].

The following risk factors for POCD were identified: age, the extent of surgery, duration of anesthesia, postoperative complications, education level, neuropsychiatric disorders and medication and already existing cognitive impairment [1]. From all above, patient age impact and involvement on POCD occurrence was studied in large groups of adult patients who completed neuropsychological tests and questionnaires. These were applied at various time moments reported to surgery: before and after surgery, at hospital discharge and 3 months postoperatively. Classifying the subjects according to their age as young

* Correspondence to: Carmen Sircuța

E-mail: carmensircuta@yahoo.com age: range 18-39 years old, middle-aged: 40-59 years old and over 60 years old, it was observed that POCD could be detected in $36.6 \%$ in young people, $30.4 \%$ in middle aged to $41.4 \%$ in old aged subjects [3].

Defining postoperative cognitive dysfunction: first of all it must not be confused with postoperative delirium which is a transient disorder with fluctuant conscious state and which occurs very nearly to postoperative period. While this entity is a transient change, POCD is a persistent change in cognitive performance which can be objectively demonstrated by comparing pre and postoperative acquired scores in certain type of tests [4].

This change is common at the time of discharge from hospital in adults of all ages having undergone a major surgery, with a significant and greater risk for long-term problems in population above 60 years old [3].

\section{Objective}

The main objective of our study was to analyse postoperative neurocognitive dysfunction development and incidence in patients with cardiac and general, noncardiac surgery.

Second objectives were to determine cognitive areas involved in POCD pathology, by applying pre- and postoperative evaluation questionnaires, and also to determine the role of anesthesia and surgery type POCD etiology. 


\section{Materials and methods}

We performed a prospective observational study in the following two clinics: Clinic of Intensive Care Unit in Tirgu Mures Emergency County Hospital and Tirgu Mures Emergency Institute for Cardiovascular Diseases and Transplantation. The study was conducted from $1^{\text {st }}$ of June 2013 to $1^{\text {st }}$ of April 2016 on a sample of 130 patients undergoing general or heart surgery.

The study included patients undergoing general surgical noncardiac interventions, under general anesthesia and cardiac surgery on cardiopulmonary bypass under general anesthesia. They all completed the MoCA (Montreal Cognitive Assesment) questionnaires for assessment of postoperative cognitive dysfunction.

We did not included in our study patients under 18 years old, patients who suffered a gynecological, orthopedic or thoracic surgery (other than the cardiac one), nor patients whom cardiac interventions were proceeded without extracorporeal pump, patients who had been reoperated. We also excluded ones who were sedated for more than 24 hours postoperatively, those with visual disturbances and those who developed postoperative delirium or psychosis. We also excluded patients who deceased.

The study was possible following approval of the ethics committees of University of Medicine and Pharmacy of Tirgu Mures (approval dated on 5/30/2016), of Emercengy County Hospital of Tirgu Mures (approval dated on 23.09.2015) and of Tirgu Mures Emergency Institute for Cardiovascular Diseases and Transplantation (dated on 09/10/2016) and after we obtained the patient 's signed informed consent.

Subjects were divided into two groups with 65 members each. Group A had a heart surgery under general anesthesia and patients in group B undergone a noncardiac surgery also under general anesthesia.

\section{Patient management}

The multimodal balanced anesthetic technique was applied. All patients were anesthetized with the same type of anesthetic drugs from the following classes: the class of sedatives and hypnotics, benzodiazepines (same type of benzodiazepine were used) and propofol. Analgesia has been provided with the same opioids and same volatile was used. Neuromuscular blocade has been accomplished with non-depolarizing neuromuscular blocking. Postoperative sedation was provided with propofol while for postoperative analgesia same NSAIDs class and opioids were used.

All patients completed three times, after they have been explained and signed the informed consent form, the "Montreal Cognitive Assessment" (MoCA) in the following moments: preoperative, 24 hours after stopping any sedative or hypnotic drugs or other medicine acting on conscious state and 7 days postoperative.

The MoCA test is a free assessment tool designed for quickly screening for mild cognitive impairment. It evaluates the following skills: alternative coupling, visual-con- structive skills, language related processes and the ability to repeat certain concepts, verbal fluency, memory recall, attention, abstraction skills. This skill are related to correspondent areas of cognition like executive function, language area, attention and concentration field, memory, orientation, visual field, conceptual thinking and orientation.

Score interpretation: a final score of 26 points and more is considered normal. A score between 22-26 points suggests a mild cognitive dysfunction, while a score below 22 points, around the average of 16 may indicate a severe cognitive dysfunction, phenomenom of Alzheimer dementia type [5-9].

\section{Data recording}

The follow-up sheet included the next parameters: sex, BMI, the highest level of education attained, previous medical history, preoperative medication, the diagnostic, surgery type, time of surgical intervention, time of anesthesia (for those with noncardiac surgery), time of extracorporeal circulation, the ASA anesthetic risk, allergies, hospitalization in intensive care unit period, necessity of transfusion of blood products, inotropic support required, postoperative arrhythmias with their management, duration of postoperative mechanical ventilation, postoperative hemodynamic, neuropsychological, respiratory, renal, hepatic, or gastrointestinal complications, postoperative bleeding, postoperative infectious complications or other perioperative incidents.

The next column in the follow-up sheet was the scoring for MoCA questionnaire before surgery, 24 hours after any sedative-hypnotic infusion had stopped and 7 days after surgery for the following sections: visuo-spatial-executive field, naming, attention, language, abstraction capacity, delay recall, orientation and final score.

\section{Data anlayse}

Data were analysed using statistical processing programs GraphPad Prism 7.0 and Epilnfo. The established alpha value was $\alpha=0.05$ and with confidence interval of $95 \%$.

The normality of the data series was established using D'Agostino test. For comparing the data sets the Man Whitney test for nonparametric data series was used; while student $\mathrm{t}$-test for unpaired data was used in assessment of Gaussian distributions. The data series were compared using paired t-test for paired data, if normal distribution, and Wilcoxon test for non-Gaussian distributions. The differences between proportions was tested using Chi-square test. The correlations between variables were investigated by calculating the Pearson coefficient, Spearman r, depending on the normality of the given series of data, of course.

\section{The description of the study}

We compared the preoperative MoCa score versus 24 hours postoperative ones, preoperative versus 7 days postoperative and 24 hours postoperative versus 7 days postoperative as it follows, for all the cognitive domains, for each group 
of patients (group A-cardiac surgical patients and group B-noncardiac surgical patients).

After that we compared the scores for cognitive domains among patients undergoing noncardiac surgery and those with heart surgery as it follows: MoCA scoring at 24 hours after surgery in noncardiac patients versus cardiac patients, MoCA scoring at 7 day postoperatively for the same as above neurocognitive functions in noncardiac patients versus cardiac patients.

\section{Results}

Comparisons results for patients in group A-with cardiovascular surgery (see table I)

\begin{abstract}
MoCA score for the visuo-spatial executive neurocognitive domain

When we compared scores for test battery of MoCA questionnaire for patient in group $\mathrm{A}$ in visuo-spatial executive functions, we noticed that 24 hours postoperative score was lower than the preoperative ones. The difference was statistically significant, with a p value $<0.0001$. Differences between scores obtained preoperative and 7 days postoperative scoring and that between postoperative scores at 24 hours and 7 day were not statistically significant with a confidence interval of $95 \%$.
\end{abstract}

\section{MoCA scoring for naming cathegory}

When we compared the scoring for "naming" obtained preoperatively with those scored 24 hours and then 7 days postoperatively no statistically significant difference was noticed. Neither when comparing 24 hours with 7 days after the surgery. Surgery and anesthesia did not influence the capacity of naming and this neurocognitive domain.

\section{MoCA scoring for "attention"}

In case of cardiovascular patients, 24 hours postoperative score for attention was significantly lower than the preoperative one, $\mathrm{p}$ was equal with 0.0037 . It was not statistically significant lower than that scored 7 day postoperative. When 24 hours score was compared with that at 7 days the difference was not significant different.

\section{MoCA scoring for language field}

When we compared the scores for neurocognitive domain of language, we noticed that 24 hours postoperatively scoring was significantly lower than the preoperative one and than those scored at 7 days after surgery, when $p$ was equal with 0.0038 . When we compared preoperative moment with postoperative moment 7 days after surgery, the difference was not statistically different.

\section{MoCA scoring for abstract skills}

For this neurocognitive part, the score difference was statistically significant only when comparing postoperative test at 24 hours versus those at 7 days; $p$ was equal to 0.0452 .

\section{Scores for orientation}

When we compared the scores for "orientation" domain those scored 24 hours after surgery were significant lower than those scored before surgery and 7 days after, when $p$ value was 0.0018 but with no statistically significant difference before surgery and 7 days after.

\section{"Recall" neurocognitive field scores}

In this field score analyse, the difference between 24 hours and 7 days after surgical procedure was statistically significant with a $\mathrm{p}$ of 0.0043 for a confidence interval of $95 \%$ with lower score at 24 hours moment. The medium score improved 7 days later after surgery with a value of 3.541 versus 24 hours after surgery, of 2.541 .

\section{Total MoCA score}

Overall postoperative score differ in a statistically significant manner, being lower at 24 hours postoperative than the preoperative ones. These scores tend to improve 7 days after surgery, being significant higher than those at 24 hours after surgery but not being significant different than the preoperative ones.

\section{Comparisons results for patients in group B-with non- cardiac surgery (see table II)}

MoCA score for the visuo-spatial executive neurocognitive domain

Scores achieved in tests for visuo-spatial-executive neurocognitive field for patients in group B, those with noncardiac surgery, 24 hours after surgical procedure were significant lower than those preoperative ones. The difference kept up in scoring 24 hours versus 7 days postoperative, but with higher (better) scores at 7 days postoperative, with a $\mathrm{p}$ of 0.0114 . There was no statistically significant difference between preoperative and 7 days after intervention achieved scores for a confidence interval of $95 \%$.

\section{MoCA score for "naming" neurocognitive domain}

For this category the only statistically significant differences between the scores achieved were those in preoperative versus 24 hours postoperative, when $\mathrm{p}=0.0051$.

\section{MoCA score for "attention"}

For this category the only significant difference was between the postoperative scores, when that one scored at 24 hours after surgical intervention was lower than that one score after 7 days, with $\mathrm{p}=0.0025$, for the same $95 \%$ confidence interval.

\section{MoCA scoring for "language" neurocognitive domain}

When we compared the scores achieved by the patients in group B, for the neurocognitive field "language" we noticed that 24 hours postoperative scores were significant lower than the preoperative ones and $\mathrm{p}$ had a value of 0.0004. 24 hours scores was significant lower that 7 
Table I. MoCA results for group A (cardiac heart surgery patients)

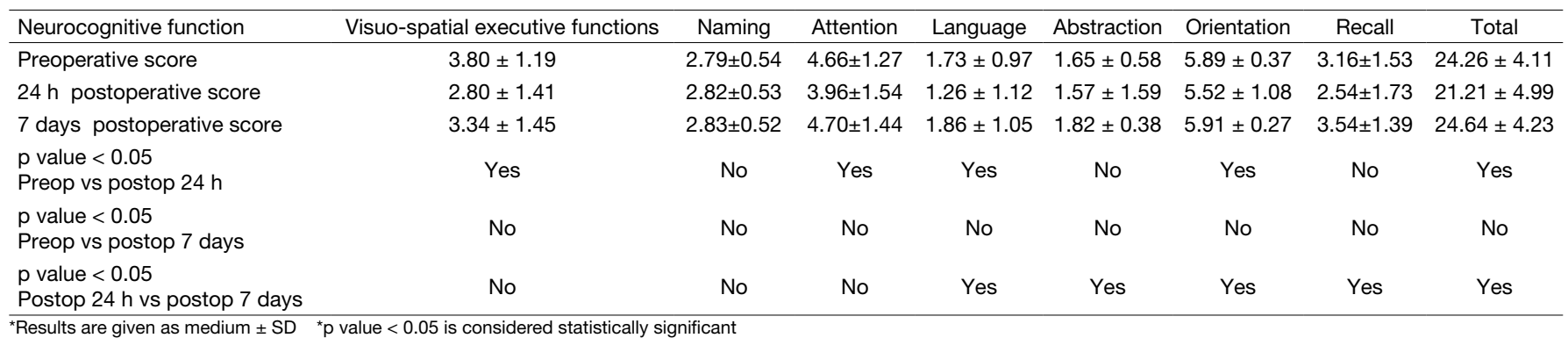

days (postoperative) scores also, when $\mathrm{p}$ was equal to 0.05 . Medium scores for preoperative test moment and that at 7 days postoperative was equal and it was of 1.738 with standard deviation of 0.973 and 1.122 and confidence interval of $95 \%$.

\section{MoCA scoring for "abstraction" neurocognitive domain}

For this category the only statistically significant difference was between preoperative and postoperative 24 hours tests when patient scored lower test value. $\mathrm{p}=0.0026$ for this comparison.

\section{MoCA scoring for "recall" neurocognitive domain}

Here the significant difference was between postoperative scores when that at 24 hours was significant lower than that at 7 days postoperative with $p$ value of 0.0219 .

\section{MoCA scoring for orientation skills}

When testing orientation skills there were no statistically significant differences between pre and postoperative scores.

\section{Total final MoCA scoring}

Total score achieved by the patients with noncardiac surgical interventions in MoCA questionnaire were significant lower when they completed the tests 24 hours after stopping any SNC active medication after surgery, than the preoperative ones. These maintained low and with significant difference versus 7 days postoperative tests, $\mathrm{p}=0.0001$. Although postoperative scoring lowers after surgery statistically significant, they tend to increase after 7 days, such as this difference is no more visible in terms of statistical significance compared with that before surgical procedure.

\section{Result of comparison of MoCA 24 hours postoperative scores between group A and B (see table III)}

Although in majority of cases (except "naming", "language" and "abstraction" categories) 24 hours postoperative scores were lower for group A, that of cardiac surgical patients, the statistically significant differences between group scores was found for the following neurocognitive fields: visuo-spatial executive domain, naming capacity, in case of abstraction skills, recall ability and orientation, where $\mathrm{p}$ value was $<0.05$ for a confidence interval of $95 \%$.

\section{Comparison results of MoCA 7 days postoperative scores, between group A and B (see table IV)}

The difference between groups maintained at 7 days postoperative also; with lower score for group A than group B in the same neurocognitive fields as for 24 hours after surgical interventions. Still the only two domains where this difference maintained statistically significant at this point after operation were visuo-spatial-executive functions and abstraction skills. There were not significant differences in total scores between two groups.

As it can be observed in the figures below (fig. 1 and fig.2), the final score was comprised, for most patients somewhere between 22 and 28 points. It showed an important decrease, higher that 2 points, sometimes as low as 20 points and below this number at 24 hours postoperatively, with a subsequent increasing at third testing, the one applied 7 days after surgery. The interesting fact is that the

Table II. MoCA results for group B ( noncardiac surgery patients)

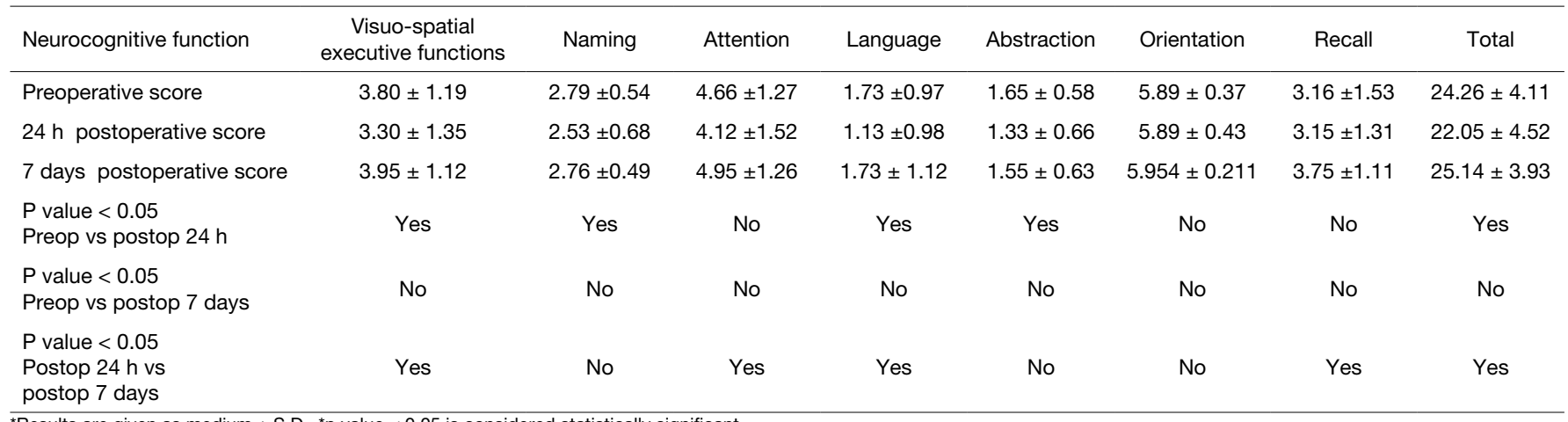

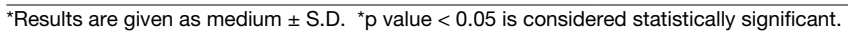


Table III. MoCA results at 24 hours testing for group A and B.

\begin{tabular}{|c|c|c|c|c|c|c|c|c|}
\hline $\begin{array}{l}\text { Neurocognitive } \\
\text { function }\end{array}$ & $\begin{array}{c}\text { Visuo-spatial } \\
\text { executive functions }\end{array}$ & Naming & Attention & Language & Abstraction & Orientation & Recall & Total \\
\hline Group A & $2.80 \pm 1.41$ & $2.82 \pm 0.53$ & $3.96 \pm 1.54$ & $1.26 \pm 1.12$ & $1.57 \pm 1.33$ & $5.52 \pm 1.08$ & $2.54 \pm 1.73$ & $21.21 \pm 4.99$ \\
\hline Group B & $3.30 \pm 1.35$ & $2.53 \pm 0.68$ & $4.12 \pm 1.52$ & $1.13 \pm 0.89$ & $0.59 \pm 0.66$ & $5.89 \pm 0.43$ & $3.15 \pm 1.31$ & $22.05 \pm 4.52$ \\
\hline$P$ value & 0.04 & 0.004 & 0.58 & 0.60 & 0.03 & 0.008 & 0.04 & 0.37 \\
\hline
\end{tabular}

Table IV. MoCA results at 7 days testing for group A and B.

\begin{tabular}{|c|c|c|c|c|c|c|c|c|}
\hline $\begin{array}{l}\text { Neurocognitive } \\
\text { function }\end{array}$ & $\begin{array}{l}\text { Visuo-spatial } \\
\text { executive functions }\end{array}$ & Naming & Attention & Language & Abstraction & Orientation & Recall & Total \\
\hline Group A & $3.34 \pm 1.45$ & $2.83 \pm 0.52$ & $4.70 \pm 1.44$ & $1.86 \pm 1.05$ & $1.82 \pm 0.38$ & $5.91 \pm 0.27$ & $3.54 \pm 1.39$ & $24.64 \pm 4.23$ \\
\hline Group B & $3.95 \pm 1.12$ & $2.76 \pm 0.49$ & $4.95 \pm 1.26$ & $1.73 \pm 1.12$ & $1.55 \pm 0.63$ & $5.95 \pm 0.21$ & $3.75 \pm 1.11$ & $25.14 \pm 3.93$ \\
\hline$P$ value & 0.01 & 0.21 & 0.29 & 0.54 & 0.01 & 0.41 & 0.54 & 0.46 \\
\hline
\end{tabular}

${ }^{\star}$ Results are given as medium \pm S.D. ${ }^{\star} p$ value $<0.05$ is considered statistically significant

average of this increase reached the preoperative scoring or even slightly above it.

In summary, the results obtained by our patient and our study were as it follows:

In case of patients of group $A$, cardiac surgical patients overall 24 hours postoperative score was lower than the preoperative one, being statistically significant lower for visuo-spatial-executive functions $(\mathrm{p}<0.0001)$, in attention testing score $(\mathrm{p}=0.0037)$, for language neurocognitive domain $(\mathrm{p}=0.0038)$, in case of orientation skills $(\mathrm{p}=0.0018)$ and for total scoring also $(\mathrm{p}<0.0001)$.

Still this postoperative decreasing of scores doesn't persist at 7 days postoperative, when score tend to have a closer value than the preoperative one. When comparing preoperative scores with those at 7 days postoperative there are no statistically significant differences.

Scoring at 24 hours after surgery is also lower than that at 7 days after surgical intervention, with significant difference for the following neurocognitive domains: language $(\mathrm{p}=0.0038)$, abstraction skills $(\mathrm{p}=0.0452)$, orientation $\mathrm{ca}-$ pacity $(\mathrm{p}=0.0018)$, recall $(\mathrm{p}=0.0043)$, and total final score $(\mathrm{p}<0.0001)$.

Patients in group $B$, those with noncardiac surgery also had an lower overall 24 hours postoperative score than that scored at the preoperative testing, with statistical significance for the next neurocognitive fields: visuo-spatial excecutive $(\mathrm{p}=0.0114)$, naming capacity $(\mathrm{p}=0.0051)$, language

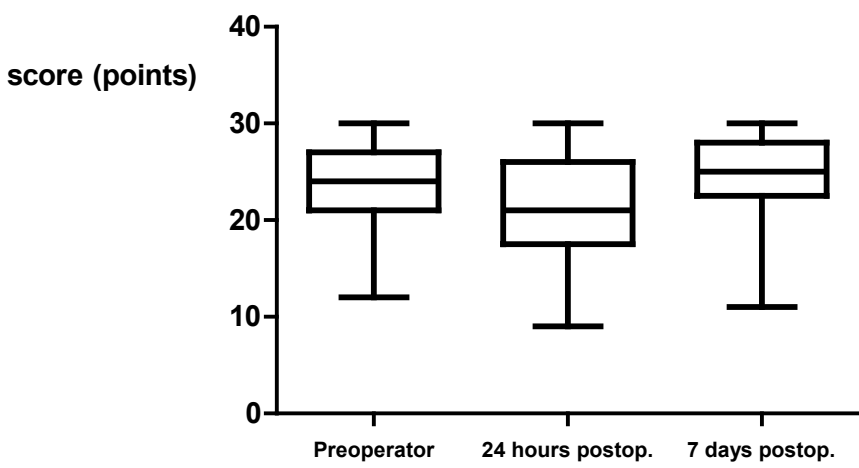

Fig. 1. Group A (cardiovascular surgery patients) results test $(\mathrm{p}=0.0004)$, abstraction skills $(\mathrm{p}=0.0026)$ and total final score $(\mathrm{p}=0.0001)$. The score improved 7 days after surgery like in group A, but with no statistically significant difference compared with the preoperative one. The increased was statistically significant when compared to the scores marked 24 hours after the procedure for the next fields: visuo-spatial executive functions $(\mathrm{p}=0.0114)$, attention $(\mathrm{p}=0.0025)$, language $(\mathrm{p}=0.0004)$, recall $(\mathrm{p}=0.0219)$ and for final score $(\mathrm{p}=0.0001)$.

24 hours postoperative score was significant lower for cardiac patients for following tested neurocognitive functions: visuo-spatial executive $(\mathrm{p}=0.0492)$, recall $(\mathrm{p}=0.0473)$ and orientation $(\mathrm{p}=0.0083)$ and statistically significant lower for grop $B$, noncardaic patients for the following fields: naming $(\mathrm{p}=0.0041)$ and abstraction $(\mathrm{p}=0.0387)$.

The difference maintained statistically significant at 7 days postoperative for visuo-spatial executive function, when group A had lower scores than group B $(\mathrm{p}=0.0143)$ and abstraction skills testing, when group $\mathrm{B}$ had lower scores than group A $(\mathrm{p}=0.0121)$.

\section{Discussions}

We chose the MoCA questionnaire, a validated 30 points test for screening even of mild cognitive impairment, with a sensivity of $90 \%$ because the older used MMSE (Mini Mental State Examination) tool is a test with low sensivity and because the majority of the adult subjects of any age score

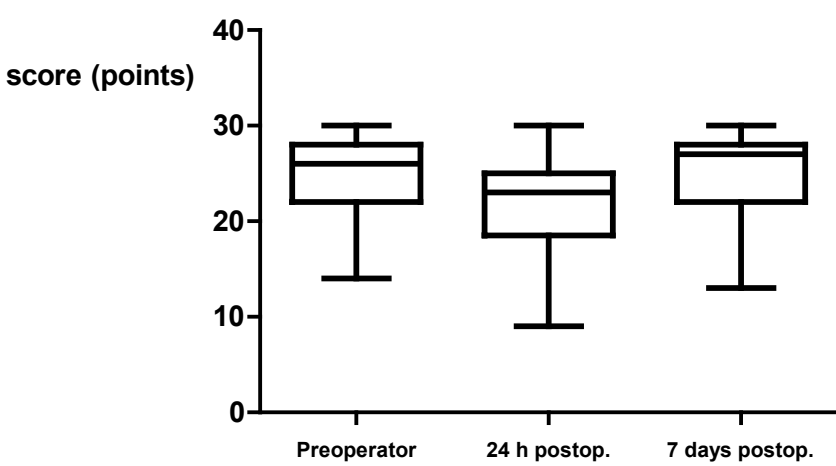

Fig. 2. Group B (noncardiac surgery) results 
very close to the maximum score and thus the minor degrees of cognitive decline are missed by applying MMSE [5, 10].

Unlike other studies [11], we did not take a cutoff/ threshold score to diagnose the emergence of POCD, since there is still no established agreement on how low the score it should be. As opinions about this vary along a cutoff point of 1,1,5, or $2 \mathrm{SD}$ (standard deviations) and is still hard to classify patients who scored lower one domain but scored more in others, we made statistical comparisons for each domain and for final score between moment before operation and after this.

Since there is no general consensus regarding the timing of assessment for POCD, and the measurements had been done beginning 1 day as long to 5 years postoperatively we chose to assess the patients at 24 hours after stopping any medication with effect on nervous system and at 7 days postoperatively. As we found no statistically significant differences at 7 days, we did not continued the investigation at 3 month after the surgical intervention. Another main reason is that in three month many external, familial and other factors have impact on patient state and may affect cognition [12].

Although the time for completing the questionnaire it was supposed to be in average 15 minutes, our subjects took in average 40 minutes to complete [13].

We also noticed an improvement in postoperative test performance in some domains which can be a learning or practice phenomenom which was also studied and described in other cited studies [14, 15].

For cardiac operation group of patients, the results of our study are different from the citated studies, with 7 days postoperative outcome showing no significant difference from the preoperative scoring with medium scores slightly higher than the preoperative ones for the majority of domains (see table 1) $[16,17]$.

We also found no statistically significant difference for noncardiac surgical group of patients between preoperative scoring and 7 days postoperative which is a different result by that shown in another study that reviewed several big databases and which found some evidence of POCD in the early period after major noncardiac surgery especially in older people [18].

A possible explanation for this difference between our study and the cited ones, could be the fact that in our research both group A and group B had a lower preoperative total score in MoCA testing than the established cutoff point for normal scoring of 26 points $[5,19]$ with a medium of 24.26 (4.111 S.D.). The cutoff point of $<25$, $<24,<23$ in MoCA scores [20] must be taken under the reserve that cutoff points must be established appropriate to culturally context and maybe that the bonus point given to those having under 12 years of schooling is just not enough to homogenize the subjects capacity of completing the questionnaire [21].

In what concerns the postoperative results for group B, noncardiac surgical patients these are not different from other studies and where was no evidence of long-term effect of cognitive decline [22].

When we take a look at domain scores we see no statisticaly significant difference for none of fields at 7 days postoperatively, neither for cardiac group, nor for noncardiac group. For noncardiac group the most affected cognitive fields at 24 hours are visuo-spatial executive, naming, language and abstraction, while for cardiac group visuospatial executive, attention, language and orientation were most affected. The comparison between the cardiac and noncardiac group showed that at 24 hours testing, group A had significant lower scores than group B for the following domains: visuo-spatial-executive, naming, abstraction, recall and orientation, while at 7 days testing the difference remains significant only for two fields like: visuo-spatialexecutive and abstraction capacity.

Since we did not find any POCD at 7 days postoperative evaluation, and since after patient is discharged many familial and external factors interfere with his/her cognition we did not consider a must, in following the patients at three months after surgery. This decision was also sustained by the low patients' compliance.

\section{Conclusions}

POCD was found at 24 four hours after surgery both in cardiac and noncardiac group. The difference was statistically significant between scores before surgical procedure versus those at 24 hours after. At 7 days postoperatively POCD was not found in any of the groups. The difference between preoperative score and that at 7 days postoperative was not statistically significant. There was no statistically significant difference in total final score between two groups at 24 hours testing nor at 7 days postoperative testing. So we can conclude that type of surgery and anesthesia did not influence the POCD emergence. Still there are some cognitive domains where POCD is present at 7 days postoperatively and this depend on type of surgery. There are statistically significant differences between the two groups, with lower score in cardiac group in 5 of 7 fields at 24 hours testing, with the persistence of difference in 2 of 7 fields.

Final conclusion of our study is the fact that we could identify different types of POCD detectable at 24 hours postoperative. Early memory and learning deficits and psychomotor slowing were reversible and disappeared in the first week in the majority of patients.

\section{Conflict of interest}

None declared.

\section{References}

1. Hovens IB, Schoemaker RG, van der Zee EA, et al. Thinking through postoperative cognitive dysfunction: How to bridge the gap between clinical and pre-clinical perspectives. Brain Behav Immun. 2012;26(7):1169-79.

2. Riedel B, Browne K, Silbert B. Cerebral protection: inflammation, endothelial dysfunction, and postoperative cognitive dysfunction. Curr Opin Anaesthesiol. 2014;27(1):89-97. 
3. Terri GM, Craig BW, Cyndi WG, et al. Predictors of Cognitive Dysfunction after Major Noncardiac Surgery. Anesthesiology 2008;108:18-30

4. Newman S, Stygall J, Hirani S, Shaefi S, Maze M. Postoperative cognitive dysfunction after noncardiac surgery: a systematic review. Anesthesiology. 2007;106(3):572-90.

5. Nasreddine ZS, Phillips NA, Bedirian V, et al. The Montreal Cognitive Assessment, MoCA: a brief screening tool for mild cognitive impairment. J Am Geriatr Soc. 2005;53: 695-699.

6. Wong GK, Lam S, Ngai K, et al. Evaluation of cognitive impairment by the Montreal Cognitive Assessment in patients with aneurysmal subarachnoid hemorrhage: prevalence, risk factors, and correlations with 3-month outcomes. J Neurol Neurosurg Psychiatry 2012;83: 1112-1117.

7. Wong GK, Wong R, Poon WS. Cognitive outcome and activity of daily living for neurosurgical patients with intrinsic brain lesions: a 1-year prevalence study. Hong Kong J Occup Ther. 2011;21: 27-32.

8. Hoops S, Nazen S, Siderowf AD, et al. Validity of the MoCA and MMSE in the detection of $\mathrm{MCl}$ and dementia in Parkinson disease. Neurology 2009;73(21): 1738-1745.

9. Oudman E, Postma A, Van der Stigchel S, et al. The Montreal Cognitive Assessment (MoCA) is superior to the Mini Mental State Examination (MMSE) in detection of Korsakoff's syndrome. Clin Neuropsychol. 2014;28(7):1123-32.

10. Hanning CD. Postoperative cognitive dysfunction. Br. J. Anaesth. 2005;95 (1):82-87.

11. Miles B, Jacob N, Jeffrey B, et al. Postoperative Cognitive Dysfunction: Minding the Gaps in our Knowledge of A Common Postoperative Complication in the Elderly. Anesthesiol Clin. 2015;33(3): 517-550.

12. Tiffany L. T, Sands LP, Leung MJ. An Update on Postoperative Cognitive Dysfunction. Adv Anesth. 2010; 28(1): 269-284.

13. An issue of anesthesiology clinics, in Brown C. Geriatric Anesthesia. Elsevier Health Sciences 2016. Pg. 520.

14. Jacobson NS, Truax P. Clinical significance: a statistical approach to defining meaningful change in psychotherapy research. J Consult Clin Psychol. 991;59(1):12-9.

15. Kevin D. Evidence-Based Indicators of Neuropsychological Change in the Individual Patient: Relevant Concepts and Methods. Arch Clin Neuropsychol. 2012; 27(3): 248-261.

16. Newman MF, Kirchner JL, Phillips-Bute B, et al. Longitudinal assessment of neurocognitive function after coronary-artery bypass surgery. $N$ Engl $J$ Med. 2001;344(6):395-402.

17. MonkTG, Weldon BC, Garvan CW, et al. Predictors of cognitive dysfunction after major noncardiac surgery. Anesthesiology 2008;108(1):18-30.

18. Newman S, Stygall J, Hirani S, Shaefi S, Maze M. Postoperative cognitive dysfunction after noncardiac surgery: a systematic review. Anesthesiology. 2007;106(3):572-90.

19. Smith T, Gildeh N, Holmes C. The Montreal Cognitive Assessment: validity and utility in a memory clinic setting. Can J Psychiatry 2007;52(5):329-32.

20. Tan JP, Li N, Gao J,et al. Optimal cutoff scores for dementia and mild cognitive impairment of the Montreal Cognitive Assessment among elderly and oldest-old Chinese population. J Alzheimers Dis. 2015;43(4):140312.

21. Koski L. Validity and Applications of the Montreal Cognitive Assessment for the Assessment of Vascular Cognitive Impairment. Cerebrovasc Dis 2013;36:6-18

22. Avidan MS, Searleman AC, Storandt M, et al. Long-term cognitive decline in older subjects was not attributable to non-cardiac surgery or major illness. Anesthesiology 2009;111(5): 964-970.

23. Wang W, Wang Y, Wu H, et al. Postoperative Cognitive Dysfunction: Current Developments in Mechanism and Prevention. Med Sci Monit. 2014; 20: 1908-1912.

24. Polunina AG, Golukhova EZ, Guekht AB, Lefterova NP, Bokeria LA. Cognitive Dysfunction after On-Pump Operations: Neuropsychological Characteristics and Optimal Core Battery of Tests. Stroke Res Treat. 2014; 2014: 302824 